\title{
Coordination of Protection and VSC-HVDC Systems for Mitigating Cascading Failures
}

\author{
R. Leelaruji, Student member, IEEE, L. Vanfretti, Member, IEEE, \\ M. Ghandhari, Member, IEEE, and L. Söder, Member, IEEE
}

\begin{abstract}
This paper proposes a methodology to coordinate protection relays with a VSC-HVDC link for mitigating the occurrence of cascading failures in stressed power systems. The methodology uses a signal created from an evaluation of the relay's status and simplifications of certain system parameters. This signal is sent to a Central Control Unit (CCU) which determines corrective action in order to reduce the risk of cascading failures.
\end{abstract}

Index Terms-Cascading failures, VSC-HVDC, Power System protection, Relay coordination

\section{INTRODUCTION}

$\mathbf{T}$ HE different synchronous systems in the European Network of Transmission system operators for Electricity (ENTSO-E) have experienced a chain of severe power system failures evidencing that these power systems are currently being operated under more stringent conditions. This fact motivates a search for methods capable of preventing severe failures or at least mechanisms to decrease the risk of blackouts. Large blackouts are the result of a complex sequence of component failures [1], equipment misoperations [2], unintended operator actions [3] and human error [4]. These complex sequence of events are commonly referred to as cascading failure or rolling blackouts. Cascading failures are rare because the most likely contingencies are considered beforehand in power system planning design and operational routines.

It can be argued that with a high degree of the "controllability" in the power system, cascading failures can be mitigated or even completely avoided. This kind of controllability is raising with the increased number of installations of FACTS devices and VSC-HVDC systems. On the other hand, protective devices commonly act as the last resource to guarantee personel and equipment safety, however under certain circumstances they might misoperate, initiating a rolling blackout.

In this paper, we hypothesize that if the operation of protective devices is coupled to the potential relief capacity of FACTS and VSC-HVDC, then cascading failures can be avoided. To this aim, we propose a control strategy coupling protection systems and VSC-HVDC links that can mitigate tripping propagation of the transmission lines. The proposed control strategy is demonstrated through digital simulation studies on a small test power system, if includes protective devices and a VSC-HVDC modeled using the PowerFactory software package [5]. Simulation results show that this control strategy improves the stability of a test power system.
In order to simulate a cascading failure, this paper begins by modeling components involved in propagating failures as explained in Section II. Next, the coordination strategy between the VSC-HVDC link and the protection system is developed in Section III. In Section IV we describe the test power system that is used in this paper and cascading failure examples along with other relevant simulations in Section V. At last, conclusions are drawn out in Section VI.

\section{SYSTEM COMPONENTS MODELING}

\section{A. Protection System modeling}

The most important components involved in cascading failures are protection systems. The cascading mechanism originates after a critical component of the system has been removed from service by the operation or misoperation of protective relays. This removal gives rise to a load flow redistribution, and as a result, other remaining components might become overloaded due to additional strain. As this process repeats sequentially it weakens the network and generates further failures which are likely to give rise to a blackout that can devastate the entire system.

In this paper, our test power system is equipped with overcurrent relays, and the inverse-time characteristics of the overcurrent relays complies with IEEE Standards [6]. The pickup time of the inverse characteristic of overcurrent relays is expressed as

$$
t(I)=\frac{A}{M^{p}-B}+C
$$

where

$t(I) \quad$ is the trip time in seconds.

$M \quad$ is the $I_{\text {input }} / I_{\text {pickup }}$ ratio (where $I_{\text {pickup }}$ is the relay currer set point), greater than zero;

$A, B, C, p$ are constants determining the desired inverse characteristic

Next we modify (1) to evaluate the relay's operating condition. To this aim we investigate the physics of the induction relay. The kinetic equation of the relay's disk which determines its full travel position is:

$$
K_{I} I^{2}=m \frac{d^{2} \Theta}{d t^{2}}+K_{d} \frac{d \Theta}{d t}+\frac{\tau_{F}-\tau_{s}}{\Theta_{\max }} \Theta+\tau_{s}
$$

where $\Theta$ is the disk position, and the remaining terms are the constant relating torque to current, $K_{I}$, the drag magnet damping factor, $K_{d}$, the input current, $I$, the moment of inertia of the disk, $m$, the disk travel to contact closure, $\Theta_{\max }$, the 
initial spring torque, $\tau_{s}$, and the spring torque at the maximum travel, $\tau_{F}$. The resulting net disk torque can be expressed as

$$
\tau_{n e t}=K_{I} I^{2}-\tau_{s}
$$

where the current, $I$, can be expressed in terms of $M$ multiplied by the pickup current $I_{p}\left(I=M I_{p}\right)$. Furthermore, the net torque on the disk is zero at $M=1$, and thus (3) reduces to,

$$
\tau_{\text {net }(M=1)}=K_{I} I_{p}^{2}-\tau_{s}=0
$$

Substituting (4) into (3), the net torque can then be expressed in terms of the spring torque,

$$
\left(M^{2}-1\right) \tau_{s}
$$

By neglecting the moment of inertia of the disk and the extra spring torque when $\Theta \neq 0$, thus (2) is then simplified and rewritten as

$$
K_{I} I^{2}-\tau_{s}=K_{d} \frac{d \Theta}{d t}
$$

To obtain the disk's position we integrate (6), yielding

$$
\Theta=\int_{0}^{T_{0}} \frac{\tau_{s}}{K_{d}}\left(M^{2}-1\right) d t
$$

This simplified expression allows us to track the status of the relay. Thus the state variable of the relay, $x_{r}$, when a transmission line has been tripped can be expressed as

$$
x_{r}=\int_{0}^{T_{0}} \frac{\tau_{s}}{K_{d} \Theta}\left(M^{2}-1\right) d t=\int_{0}^{T_{0}} \frac{1}{t(I)} d t=1
$$

This means that $\Theta$ becomes $\Theta_{\max }$, and $T_{0}$ represents the time required for the disk to complete one full rotation. Hence, the integration of (8) equals unity. This condition, $x_{r}=1$, forces the relay logic to order the circuit breaker to trip the transmission line.

According to NERC's transmission relay loadability standard [7], overloading capacity must be included in the system in order to account for errors that might result in overtripping. These errors include voltage variation due to shortterm transmission line overloading, transient overreach, or unspecified inaccuracies. In other words, transmission lines could be overloaded within a certain range in order to prevent unnecessary relay operations. Under these considerations we modify (8) to obtain a new expression of the relay's internal state variable. In addition according to the loadability standard, a relay should pickup when current is at least $15 \%$ higher than pre-defined pickup current. In this paper VSC-HVDC link is coordinated with protective relay's so that a certain relief is provided to the system. To this aim we select the relay to pick up when the current is $20 \%$ higher than the pickup current, thus providing more opportunity for the VSCHVDC to support the system. Hence the relay's state variable is calculated from

$$
x_{r}= \begin{cases}\int_{0}^{T_{0}} \frac{1}{t(I)} d t & ;|I| \geq 1.2 I_{p}, \\ 0 & ;|I|<1.2 I_{p}\end{cases}
$$

\section{B. Load Modeling}

Dynamic load models provide a more accurate description of the load behavior in realistic power systems than static load models. Hence, we have chosen a non-linear dynamic load model with exponential recovery, which is given by [8]

$$
\begin{aligned}
\frac{d x_{p}}{d t} & =\frac{1}{T_{L p}}\left(-x_{p}+P_{s}\left(V_{L}\right)-P_{t}\left(V_{L}\right)\right) \\
P_{L} & =x_{p}+P_{t}\left(V_{L}\right)
\end{aligned}
$$

where $x_{p}$ is the state accounting for the active load recovery dynamics. The active load model is parameterized by the steady-state voltage dependency $P_{s}(V)=P_{0} V^{\alpha_{s p}}$, the transient voltage dependency $P_{t}(V)=P_{0} V^{\alpha_{t p}}$, and a recovery time constant $T_{L p}$. $P_{L}$ represents the actual active power load while $P_{0}$ is the sum of the rated power of the connected load. The equation for reactive power load follows the same form, and thus it is not included here. The steady-state voltage dependency quantifies how much load has been restored after the recovery. In this paper, $\alpha_{s p}$ is equal to zero which means a fully restored load and $\alpha_{t}$, in the transient load-voltage dependence term, is equal to 2 which means that the load has a constant impedance characteristic.

\section{VSC-HVDC modeling}

In this paper the VSC-HVDC is modeled using an injection model similar to the one described in [9], which states that the production or consumption of active power is independent of the production or consumption of reactive power. This means that the VSC-HVDC link can be modeled using controllable $\mathrm{AC}$ voltage source as shown in Fig. 1. The injected active

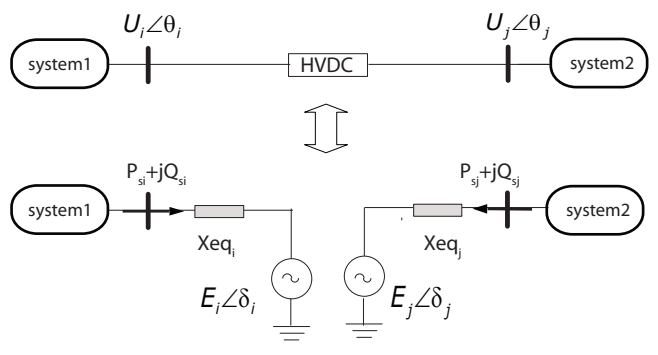

Fig. 1. Simplified model of VSC-HVDC link

power can be calculated as follows,

$$
\begin{gathered}
P_{s i}=\frac{U_{i} E_{i}}{X_{e q_{i}}} \sin \left(\theta_{i}-\delta_{i}\right) \\
P_{s j}=\frac{U_{j} E_{j}}{X_{e q_{j}}} \sin \left(\theta_{j}-\delta_{j}\right)
\end{gathered}
$$

and the injected reactive power can be expressed as

$$
\begin{gathered}
Q_{s i}=\frac{U_{i}\left[U_{i}-E_{i} \cos \left(\theta_{i}-\delta_{i}\right)\right]}{X_{e q_{i}}} \\
Q_{s j}=\frac{U_{j}\left[U_{j}-E_{j} \cos \left(\theta_{j}-\delta_{j}\right)\right]}{X_{e q_{j}}}
\end{gathered}
$$


where $P_{s i}=-P_{s j}$. The voltage source can be controlled by manipulating the magnitude of $E_{i}$ and the phase angle $\delta_{i}$. This is done by explicitly expressing the complex power as

$$
\begin{aligned}
\bar{S}_{s i} & =P_{s i}+j Q_{s i} \\
& =\frac{U_{i} E_{i}}{X_{e q_{i}}} \sin \left(\theta_{i}-\delta_{i}\right)+\frac{U_{i}\left[U_{i}-E_{i} \cos \left(\theta_{i}-\delta_{i}\right)\right]}{X_{e q_{i}}}
\end{aligned}
$$

Thus,

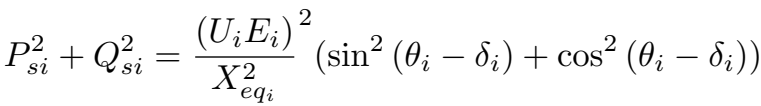

$$
\begin{aligned}
& -\frac{2 U_{i}^{3} E_{i}}{X_{e q_{i}}^{2}} \cos \left(\theta_{i}-\delta_{i}\right)+\frac{U_{i}^{4}}{X_{e q_{i}}^{2}} \\
& =\frac{U_{i}^{4}-2 U_{i}^{3} E_{i} \cos \left(\theta_{i}-\delta_{i}\right)+\left(U_{i} E_{i}\right)^{2}}{X_{e q_{i}}^{2}}
\end{aligned}
$$

Rewriting (11a) and (12a), the difference in the phase angles can be expressed as

$$
\begin{aligned}
& \sin \left(\theta_{i}-\delta_{i}\right)=\frac{P_{s i} X_{e q_{i}}}{U_{i} E_{i}} \\
& \cos \left(\theta_{i}-\delta_{i}\right)=\left(Q_{s i}+\frac{U_{i}^{2}}{X_{e q_{i}}}\right) \frac{X_{e q_{i}}}{U_{i} E_{i}}
\end{aligned}
$$

Substituting (16) into (14), $E_{i}$ can be computed as follows

$E_{i}=\frac{1}{U_{i}} \sqrt{\left(P_{s i}^{2}+Q_{s i}^{2}\right) X_{e q_{i}}^{2}-U_{i}^{4}+2 X_{e q_{i}} U_{i}^{2}\left(Q_{s i}+\frac{U_{i}^{2}}{X_{e q_{i}}}\right)}$

From (15), the phase angle $\delta_{i}$ can be computed as

$$
\delta_{i}= \begin{cases}\theta_{i}-\arcsin \left(-\frac{P_{s i} X_{e q_{i}}}{U_{i} E_{i}}\right) & ; \cos \left(\theta_{i}-\delta_{i}\right)>0, \\ \theta_{i}-\left[\pi-\arcsin \left(-\frac{P_{s i} X_{e q_{i}}}{U_{i} E_{i}}\right)\right] & ; \cos \left(\theta_{i}-\delta_{i}\right)<0\end{cases}
$$

where $P_{s i}$ and $Q_{s i}$ are determined as explained in Section III. The phase angle, $\delta_{i}$, and the voltage magnitude, $E_{i}$ are used to control he output power from the VSC-HVDC.

\section{COORDINATION OF PROTECTION AND CONTROL SYSTEMS}

The proposed coordination algorithm between the protection system and the injection model of the VSC-HVSC link is shown in Fig. 2. This control strategy uses the $x_{r_{i}}$ states from all transmission lines, and other measurements, to determine the active power modulation of the VSC-HVDC. An overcurrent relay installed on each transmission line generates a value $x_{r_{i}}$ that is responsible for creating the activation signal that modulates the injected power from the voltage source. The current signal, $I$, is used in this control algorithm because the operational logic of the overcurrent relays uses only this variable for monitoring. The $x_{r_{i}}$ values from each relay are sent to a Central Control Unit (CCU) in order to determine a maximum value, $x_{\max }$, among all connected transmission lines. Recalling from (8) that the relay triggers the breaker to trip if $x_{r}$ equals unity, the value $x_{\max }$ indicates the most susceptible line to be tripped which might avoid a cascading failure.

Once $x_{\max }$ is determined at the $\mathrm{CCU}$, is then compared with the operating setpoint, $x_{r e f}$. This $x_{r e f}$ is a pre-defined value which determines the activation of VSC-HVDC. If $x_{\max }$ is larger than $x_{r e f}$, then the voltage source is activated. The active power modulation $\left(P_{s i}\right)$ of the voltage source is determined by a PI-controller, which uses $I_{p}$ and $I$ as inputs. The pickup current, $I_{p}$, has been pre-defined, and the current magnitude, $I$, corresponds to the line which has the highest $x_{r}$. Meanwhile, the modulation of reactive power $\left(Q_{s i}\right)$ in this paper is kept at zero.

\section{Test Power System}

The test power system used in the simulations of this section is the Single-Load-Infinite-Bus (SLIB) system shown in Fig. 3. This simple model is a conceptualization of the most relevant characteristic of the Swedish Grid: generation in the North and consumption in the South. The generation area is represented by an infinite bus, and the load area is represented by the equivalent non-linear dynamic load model described in Section II-B. Occasionally, voltage-dependent characteristics have negative impacts on the power system due to the possible development of slow voltage instability, which might lead to blackouts such as the Swedish system collapse in 1983 [10]. The impedance of each transmission line is given in Table I,

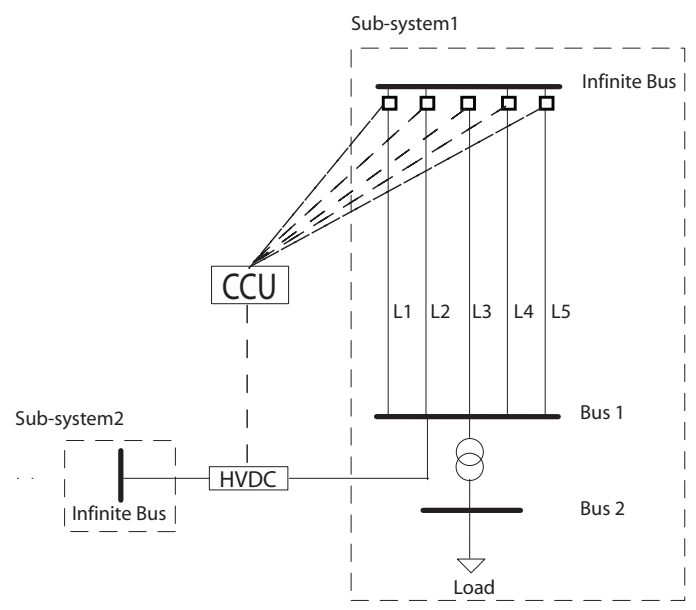

Fig. 3. Test power system

although these line parameters might not be strictly realistic for an actual power network, we have selected them so that cascading failures can be easily simulated for this test network. The voltage level at the high-voltage side (Bus 1) and the low-voltage side (Bus 2) are equal to $400 \mathrm{kV}$ and $220 \mathrm{kV}$, respectively.

TABLE I

TRANSMISSION LINE DATA

\begin{tabular}{ccc}
\hline Line & $R+j X[\Omega]$ & $B[\mu \mathrm{S}]$ \\
\hline L1 & $8.0+\mathrm{j} 80.00$ & 14.0 \\
L2 & $9.6+\mathrm{j} 96.00$ & 16.8 \\
L3 & $14.4+\mathrm{j} 144.00$ & 25.2 \\
L4 & $18.4+\mathrm{j} 184.00$ & 32.2 \\
L5 & $8.0+\mathrm{j} 80.00$ & 14.0 \\
\hline
\end{tabular}

The parameters of the non-linear dynamic load described in Section III are defined in Table II. 


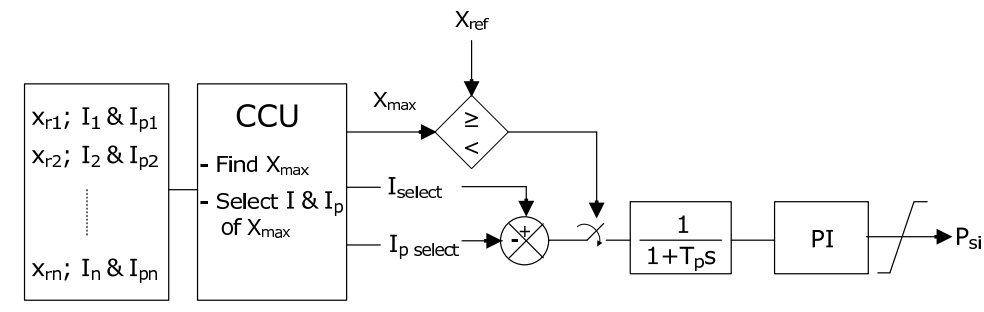

Fig. 2. Coordination of the protection relays and the VSC-HVDC injection model

TABLE II

LOAD PARAMETERS

\begin{tabular}{rllrlcc}
\hline Active Power & Reactive Power & Unit \\
\hline$P_{0}=$ & 160 & $Q_{0}$ & $=$ & 50 & MW \\
$T_{L p}=$ & 20 & $T_{L q}$ & $=$ & 20 & sec \\
$\alpha_{s p}$ & $=$ & 0 & $\alpha_{t p}$ & $=$ & 2 & - \\
$\alpha_{s q}$ & $=$ & 0 & $\alpha_{t q}$ & $=$ & 2 & - \\
\hline
\end{tabular}

The PI-controller shown in Fig. 2 is given by

$$
\mathrm{PI}=K_{p}+\frac{K_{i}}{s T i}
$$

where the controller parameters are defined as $K_{p}=4$, $K_{i}=3.5, T_{i}=1 \mathrm{sec}$, and $T_{p}=1 \mathrm{sec}$. This parameters have been selected for illustration purposes. In this paper we have limited our investigation to the development of the control strategy. Thus, the important issue of appropriate compensation design using the PI-controller which drives the VSC-HVDC modulation is not addressed here. We realize that proper controller design methods should be used so that the full potential for relief available from the VSC-HVDC can be exploited while satisfying different design specifications and considering physical limits of the devices.

The capacity of the VSC-HVDC considers a restricted operation area explained in [11], which does not account for the dc cable and dc voltage limitation: i.e. $S^{2}=P^{2}+Q^{2}$. In addition, all VSC-HVDC losses are neglected. The parameters for the overcurrent relays in (1) are selected from the standard time-current characteristics in [6], and listed in the Table III.

TABLE III

RELAY PARAMETERS

\begin{tabular}{ccccc}
\hline Characteristic & A & B & C & p \\
\hline Very inverse & 19.61 & 1 & 0.491 & 2 \\
\hline
\end{tabular}

\section{Case Studies}

In this section we present several simulations that illustrate the control strategy discussed in Section III. In these simulations we have considered the loss of transmission lines as the contingencies which could lead to a cascading failure. We simulate several scenarios which include the loss of multiple lines with and without the inclusion of our coordination strategy.

\section{A. Case 1: One line tripped - without coordination}

In this case, transmission line L1 is tripped at time $t=1$

S.
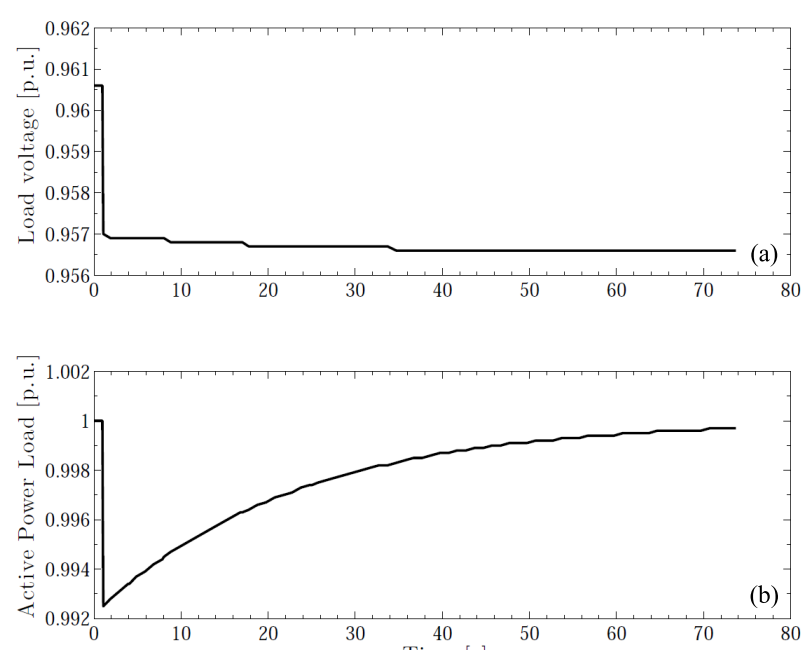

Fig. 4. Active power consumed by the load (a) and load voltage profile (b)

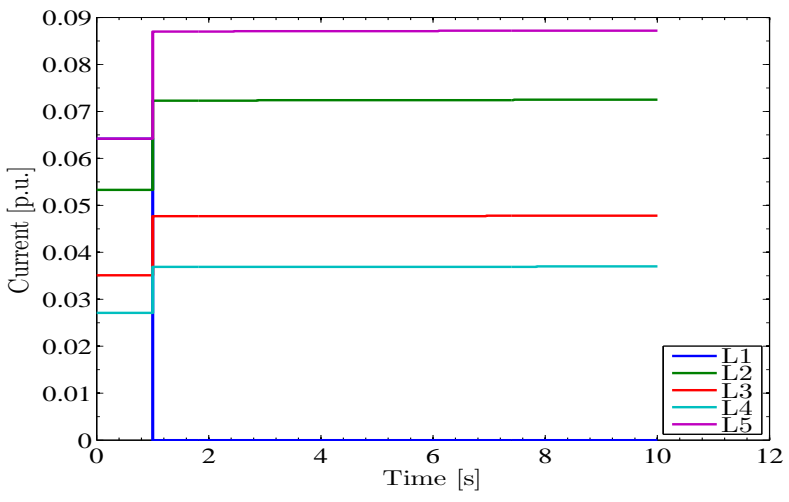

Fig. 5. Line current profile after L1 is tripped

As shown in Fig. 4, the voltage at load bus drops from 0.9606 p.u. to 0.9566 p.u. However, because of the non-linear dynamic load model, the active power load increases to its nominal value regardless of the voltage drop at the load bus. Figure. 5 shows the current flow increase within the remaining lines, which a reflection of the load flow re-distribution when line L1 is tripped. However, the value of these currents remains below the trigger point which would activate the relays.

\section{B. Case 2: Two lines tripped - without coordination}

This simulation case continues from Case 1, where L1 is tripped at $t=1 \mathrm{~s}$, and L5 is tripped at $t=4 \mathrm{~s}$. The load 
flow redistribution increases the current through L2 until it tripped at $t=13.881 \mathrm{~s}$. Consequently, L3 and L4 tripped at $t=19.026 \mathrm{~s}$, and $t=19.979 \mathrm{~s}$, respectively (as shown in Fig. 7). The protection logic used to trip the lines uses the internal state variable of each relay, $x_{r_{i}}$. If any of the $x_{r_{i}}$ is greater than $x_{r} e f$, then the relay sends a signal to the circuit breaker in order to trip the line, as shown in Fig. 8. The simulation was performed for only $20 \mathrm{~s}$, and Fig. 6 shows the voltage at the load bus and the active power load at $0.01 \mathrm{~ms}$ before the system collapse.
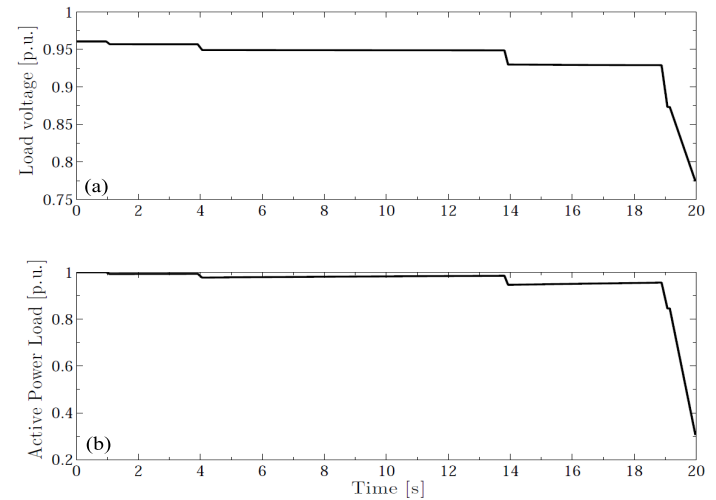

Fig. 6. Load bus voltage (a) and load response (b) - without control

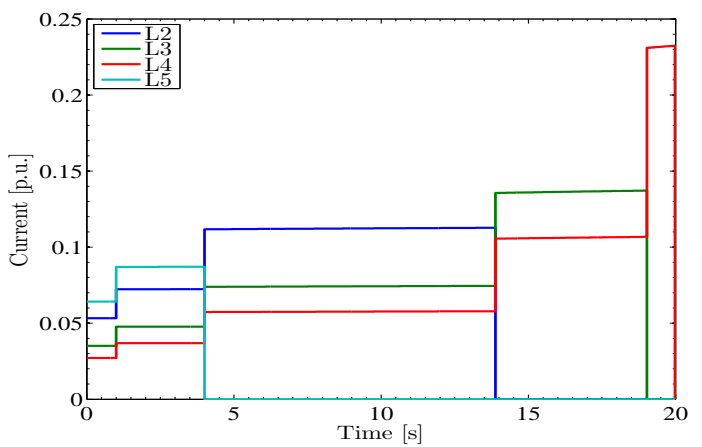

Fig. 7. Line current profile after L1 and L5 are tripped - without control

Fig. 8 also shows $x_{\max }$, which can be used to indicate which line will be put out of service. The left-most peak, when $x_{\max }$ reaches one, corresponds to the time when L2 is tripped, the middle peak corresponds to the time when line L3 is tripped and finally, the right-most peak represents the time when L4 is tripped. Next we illustrate how this variable can be used to coordinate the protection relays and the VSC-HVDC.

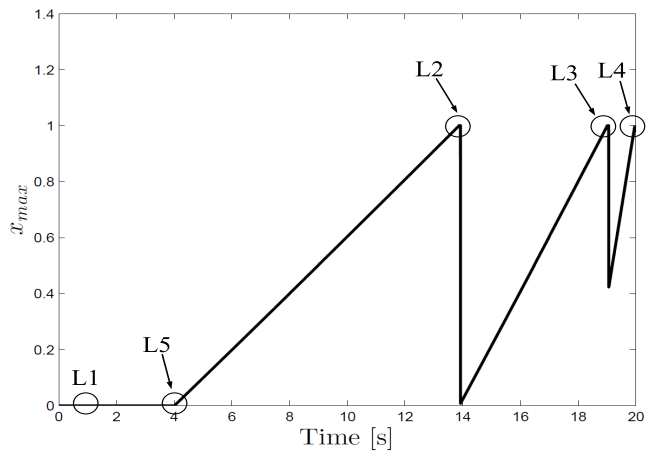

Fig. 8. Maximum relay state variable - without control

\section{Case 3: Two lines tripped - with coordination}

In this section we illustrate the control strategy proposed in Section III. To this aim, we present three different cases of power injection. In Case 3.1: Infinite Capacity, the capacity of VSC-HVDC is unlimited. In Case 3.2: Limited Capacity, the capacity of VSC-HVDC is equal to 45 MVA which is one-third of the active power load. And finally, Case 3.3: Minimum Injected Power, the VSC-HVDC is controlled to inject the minimum required power to prevent a cascading failure. Fig. 9 compares of $x_{\max }$ between Case 2 and Case 3.1 which includes the control of the VSC-HVDC link. The figure shows that by controling the VSC-HVDC, $x_{\max }$ returns to zero before L2 is tripped. The responses of $x_{\max }$ are similar for Case 3.2 and 3.3. In addition, as shown in Fig. 10, with power injection, the current passing through each line decreases.

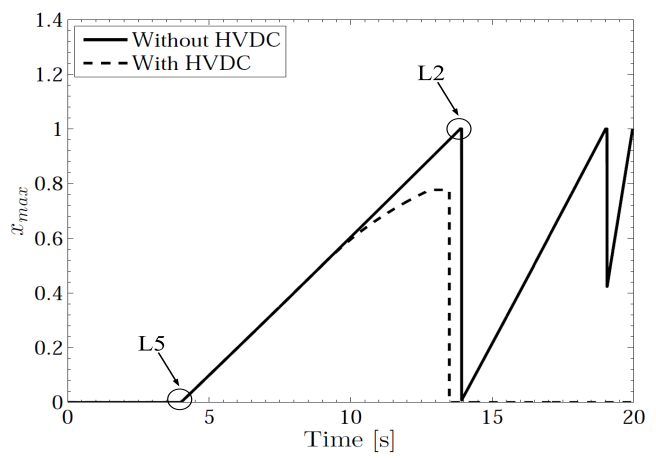

Fig. 9. Comparison of $x_{\max }$ between system with and without VSC-HVDC control

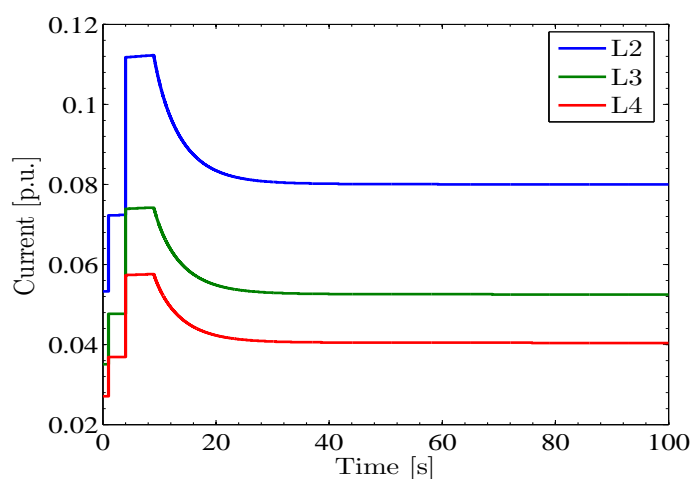

Fig. 10. Line current profile after L1 and L5 are tripped

Fig. 11 shows the injected power for Cases $3.1-3.3$, Fig. 12 shows the current in L2 for all cases and Fig. 13 shows the voltage and active power at the load bus. All cases are successful in preventing the cascading failure.

In Case 3.1, the current is restored to the pickup current level of the relay $\left(I_{p}\right)$, by the use of the VSC-HVDC. Note that the active and reactive power output of he VSC-HVDC have not been limited here. In Case 3.2, although the current was not be able to return to the pickup current level $\left(I_{p}\right)$, the support from the VSC-HVDC was be able to decrease it below the overloading capacity $\left(|I|<1.2 I_{p}\right)$. Note that 
this is a result of limiting the capacity of the VSC-HVDC. In Case 3.3, we investigate what is the minimum power necessary to keep $x_{\max }$ below one at all times. In other words, constant power is injected and $x_{\max }$ returns to zero and the injected power is set to prevent additional line tripping.

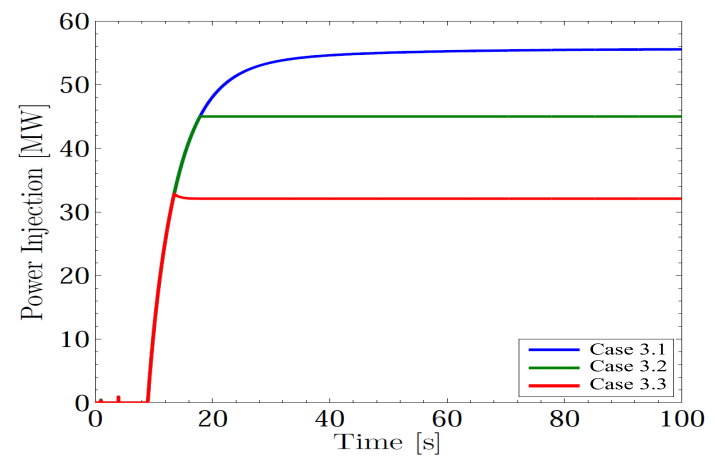

Fig. 11. Active power injected for three cases

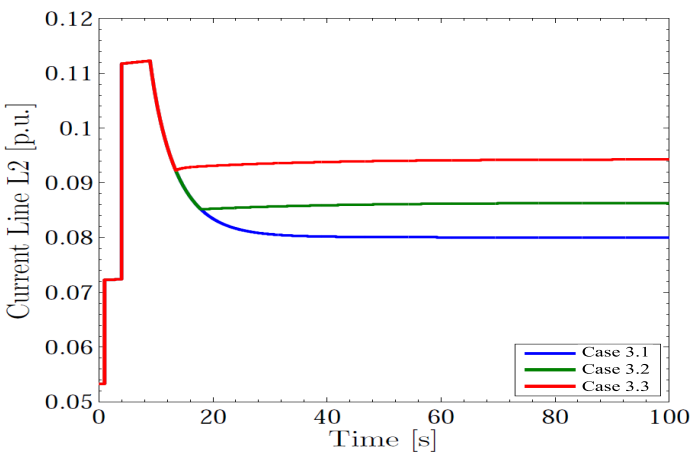

Fig. 12. Current in Line L2 for three cases
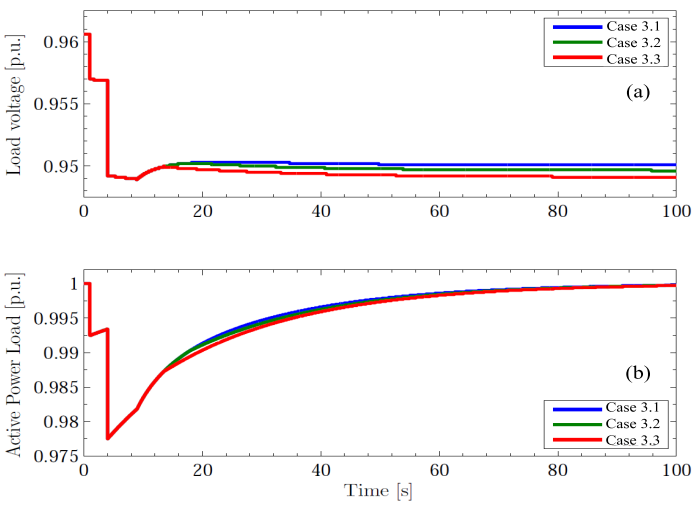

Fig. 13. Load bus voltage (a) and load response (b) for three cases

It should be noted that the response of the VSC-HVDC is controlled by the PI-controller discussed in Section III. We realize that with more appropriate tunning the response of the VSC-HVDC could be fast enough so that the cascading failure is avoided and that the system strain is more effectively relieved. For example, in some circumstances a faster response of the VSC-HVDC could limit the tripping at relays which are beyond the the pickup level as in Case 3.2. To illustrate the importance of proper tunning, Fig. 14 shows the injected power of the VSC-HVDC for different values of $K_{i}\left(K_{p}\right.$ is kept constant and equal to 4) observe that under different controller parameters a faster response of the VSC-HVDC can be obtained. Also note that for this purpose, design requirements such as rise time, overshoot, time-to-peak, and steady-state error have to be considered, while at the same time respecting physical limits are VSC-HVDC. In this paper we have focused on the control strategy, an the important issue of control tunning will be addressed in a future publication.

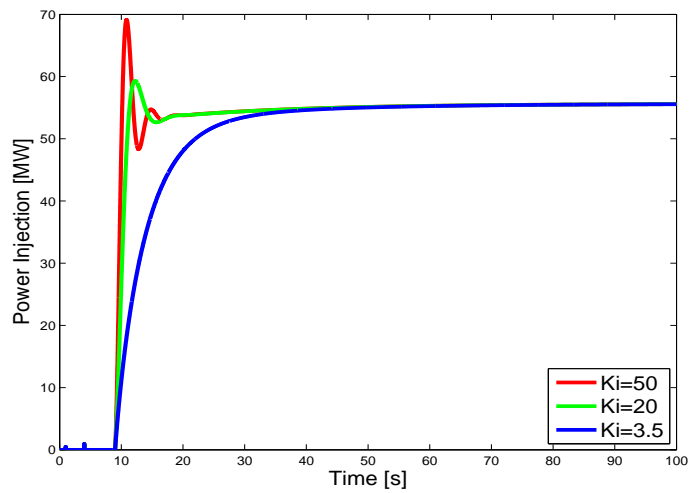

Fig. 14. Injected power for different $K_{i}\left(K_{p}=4, T_{i}=1\right)$

\section{Case 4: Three lines tripped - effect of selected $x_{r e f}$}

This simulation Case is an extension of Case 3.3 where two lines are tripped. Here a third-line (L3) is tripped for three different scenarios, which are:

Case 4.1: Tripping of L3 after the initial power injection of the VSC-HVDC link $\left(x_{r e f}=0.5\right)$ : In this case, L3 is tripped at $t=16.5 \mathrm{~s}, 3 \mathrm{~s}$ after the power injected reaches its minimum required level for preventing a cascading failure. The line trips after $x_{\max }$ returns to zero. Subsequently, $x_{\max }$ increases, requiring more power injection. Fig. 15 shows when the minimum required power reaches, $P=89 \mathrm{MW}$. This means that in order to secure the system, the rating of VSCHVDC has to be capable of providing this amount of power injection. The middle peak in Fig. 15(a) starts to rise when L3 is tripped. At the meantime, more active power is injected to avoid the tripping of the other lines. Due to the power injection, this middle peak stops increasing near to 0.99 before returning to zero. The right-most peak occurs as a result of the active power load recovery. Because of this load increment, the current in the lines is increased beyond their overload capacity. As a consequence, $x_{\max }$ raises. When $x_{\max }$ reaches 0.5 , the power injection increases from $85 \mathrm{MW}$ to $89 \mathrm{MW}$, and this injection forces $x_{\max }$ to return to zero again.

Case 4.2: Tripping of L3 before the initial power injection of the VSC-HVDC link $\left(x_{r e f}=0.5\right)$ : In this case, L3 is tripped at $t=10.5 \mathrm{~s}$ which is $3 \mathrm{~s}$ before the injected power reaches its minimum required level for preventing a cascading failure. Here, the third line is tripped while the variable $x_{\max }$ is increasing. Fig. 16 shows that at $t=10.5 \mathrm{~s}$, the slope of $x_{\max }$ becomes steeper. This means that the line will be tripped sooner compared to the previous case. On the other hand, the VSC-HVDC injects active power in accordance with the change of $x_{\max }$. Unfortunately, line L2 is tripped before the power reaches to the new minimum required value. Consequently, all the lines are tripped as shown in Fig. 17. 

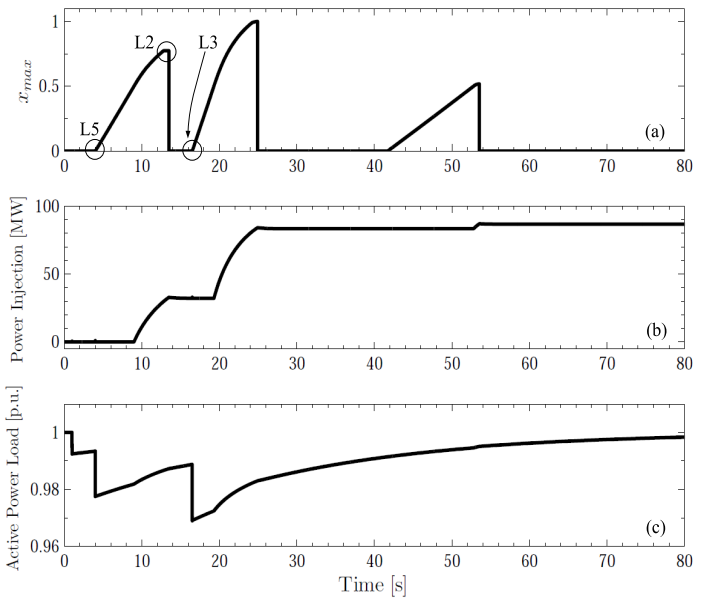

Fig. 15. Case 4.1: $x_{\max }$ (a), injected power (b) and load response (c)
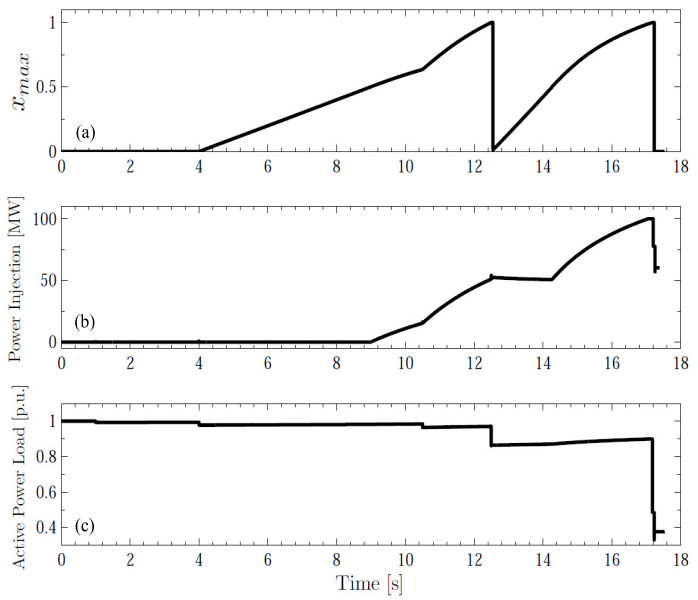

Fig. 16. Case 4.2: $x_{\max }$ (a), injected power (b) and load response (c)

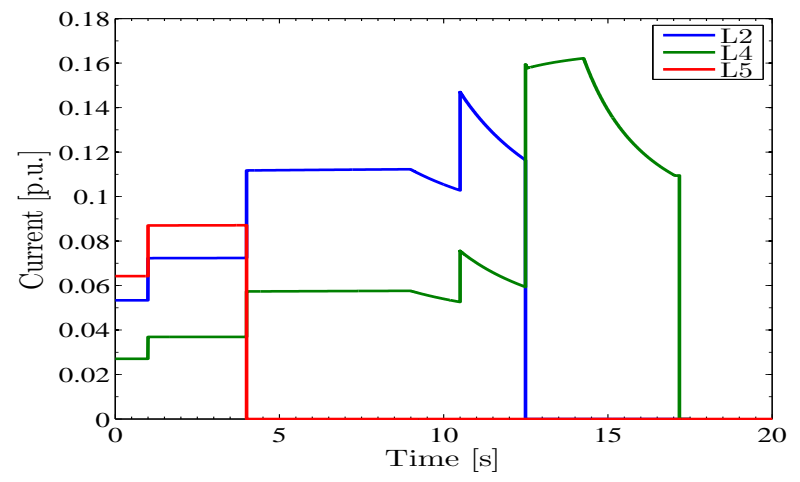

Fig. 17. Case 4.2: Line current profile

Case 4.3: Tripping of $L 3$ before the initial power injection of VSC-HVDC link $\left(x_{r e f}=0.15\right)$ : In this case, the activation level for power injection is lowered from $x_{r e f}=0.5$ to $x_{r e f}$ $=0.15$. With 2 lines tripped and the new level of $x_{r e f}$, the injected power reaches its minimum required level at $t=10.5$ $\mathrm{s}$. Thus the line L3 is tripped at $t=7.5 \mathrm{~s}$ which is $3 \mathrm{~s}$ before the injection is completed. Figure. 18 shows that the VSCHVDC is activated sooner compared to Case 4.2 , which results in a large power injection from the VSC-HVDC. The middle and the right-most peak of $x_{\max }$ in Fig. 18(a) occur due to the active power load recovery, as discussed in Case 4.1.
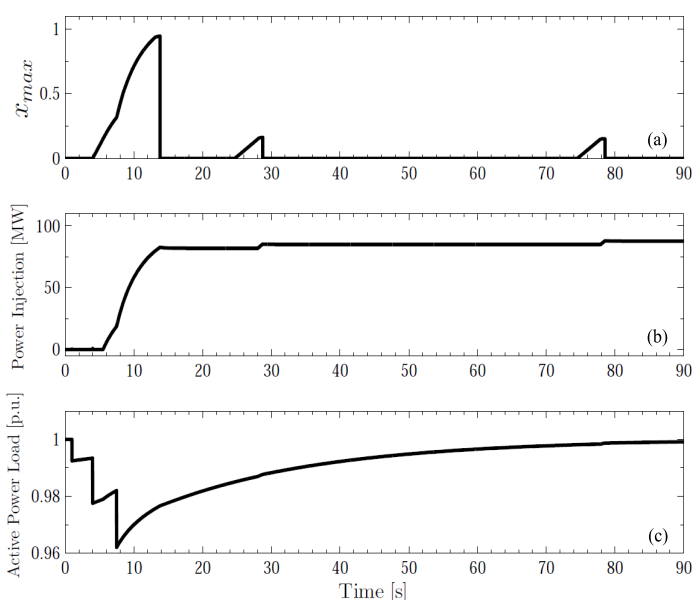

Fig. 18. Case 4.3: $x_{\max }$ (a), injected power (b) and load response (c)

From the simulation in this section, it can be concluded that in order to prevent cascading failures, our control strategy requires that $i$ ) the capacity of VSC-HVDC has to be large enough to compensate for the lost power which was transmitted through the faulted lines, and ii) with fixed PI-controller parameters, the value of $x_{r e f}$ has to be set low enough to allow time for VSC-HVDC to inject minimum required power.

\section{E. Case 5: Tripping of Lines L1, L3, and L5 - Comparison of VSC-HVDC}

In this section, the VSC-HVDC described in Section II-C, is compared with the built-in model from PowerFactory. The simulation is performed according to the setting described in Case 4.3. The active power set point in the built-in model uses the same control block diagram as the one in Fig. 2. The builtin model also neglects no-load losses, copper losses, and short circuit impedance. Fig. 20(b) shows that the built-in model injects power in the higher rate. As a result, $x_{\max }$ in Fig. 20(a) for the built-in model reaches only 0.6, whereas the injection model reaches approximately 0.9 . This suggests that the builtin model can be activated later in order to prevent cascading failure, compared with the injection model. The active power load response shown in Fig. 20(c) and the current in L2 in Fig. 19(a) are similar for both models. In Fig. 19(b) the voltage at the load bus is also similar for both models which is dropped approximately from 0.96 to 0.94 . From this comparison it is possible to argue that the injection model of the VSC-HVDC is suitable for cascading failure simulations.

\section{F. Case 6: $P Q$ versus $P V$ control}

The simulation in this section is performed using only the VSC-HVDC built-in model, all setting are the same as in Case 4.3. The control mode of VSC-HVDC is changed from PQ control (where Q has been set to zero) to PV control. This means that the voltage at the bus where VSC-HVDC is connected must be kept at the same level it was during pre-disturbance conditions (see Fig. 22). Figure. 21 shows that in PV mode, the active power injection reduces from 89 MW to $62 \mathrm{MW}$ and the voltage level is compensated by the injection of reactive power that is changed from zero to 48 MVAR. In addition the minimum rating of VSC-HVDC that 

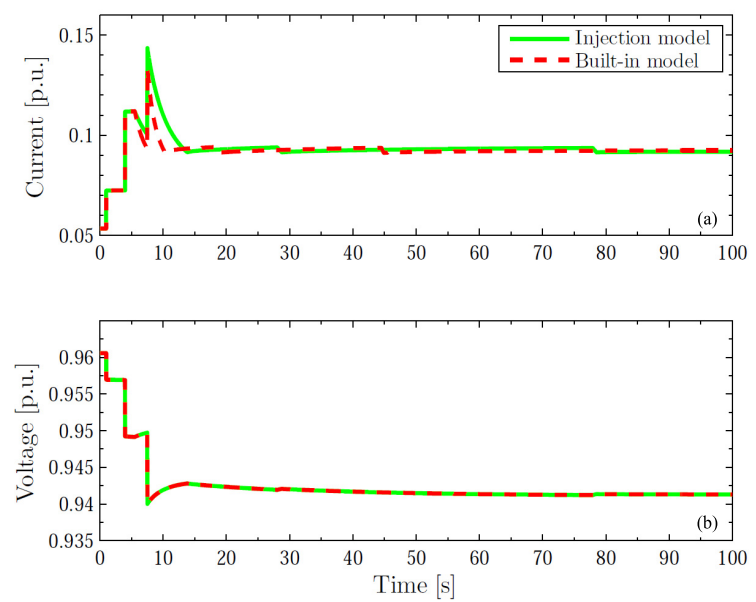

Fig. 19. L2 current (a) and Load bus voltage (b)
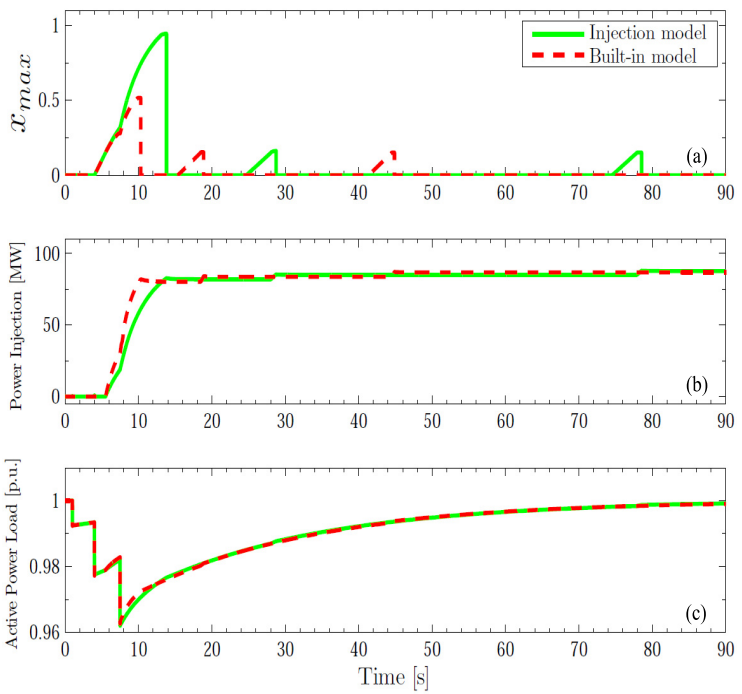

Fig. 20. $x_{\max }$ (a), Injected power (b) and Load response (c)

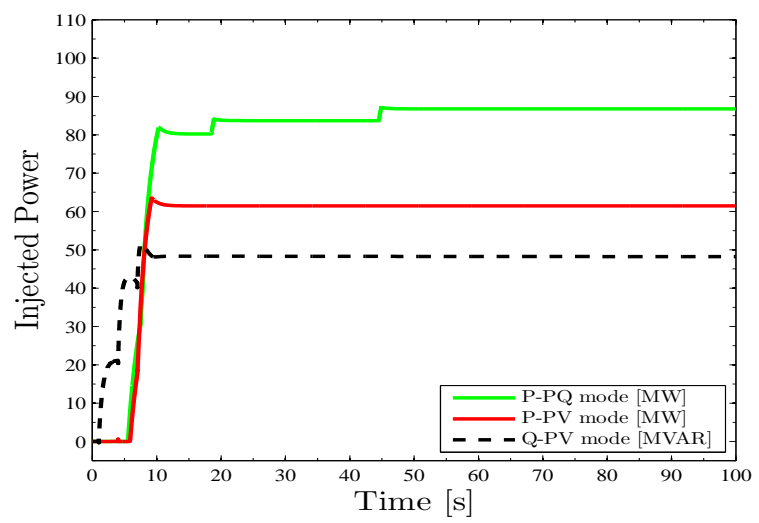

Fig. 21. Injected power - PQ mode versus PV mode

is required to avoid system collapse decreases from 89 MVA to approximately 79 MVA.

From this case study, it can be concluded that both control modes can be used to prevent the system from cascading failure. The selection of the control mode depends on the rating of the VSC-HVDC and also on the voltage drop at the load bus. If the voltage drop is lower than acceptable level in the system, the PV mode is more suitable.

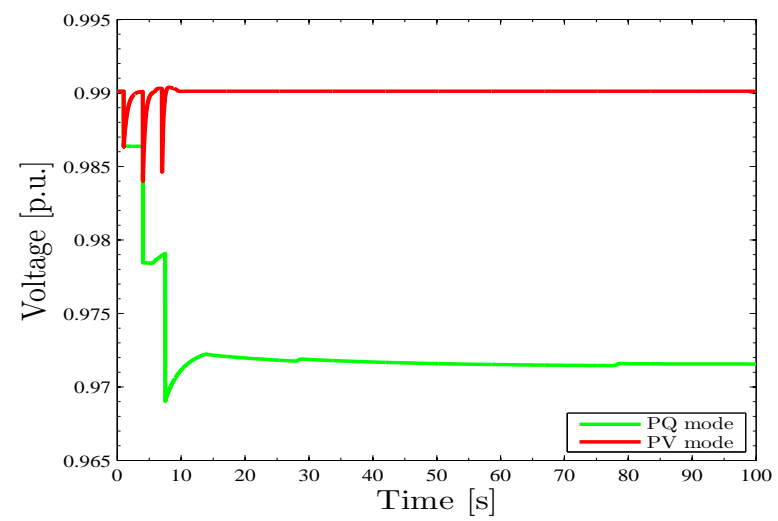

Fig. 22. Voltage at Bus 1 - PQ mode versus PV mode

\section{CONCLUSIONS}

This paper has explored how cascading failures can be mitigated through the coordination of protection systems and VSC-HVDC links. By mean of simulation studies, a proposed control strategy for VSC-HVDC links and protection systems coordination was illustrated. The control strategy uses signals from protective relays and from the VSC-HVDC bus. By simulating different contingencies, we have shown that with proper coordination of the relays and VSC-HVDC, the VSCHVDC is successful in decreasing the current in all remaining transmission lines and supporting the voltage level. Thus, it suggested that the coordination of protection and VSC-HVDC can substantially reduce the risk of a cascading failures.

\section{REFERENCES}

[1] S. Larsson and E. Ek, "The blackout in Southern Sweden and Eastern Denmark, September 23, 2003," in IEEE Power Engineering Society General Meeting, vol. 2, pp. 1668-1672, 2004.

[2] U. D. of Energy, "U.S.-Canada power system outage task force. (2004) Final Report on the August 14, 2003 Blackout in the United States and Canada: Causes and Recommendations." https://reports.energy.gov/BlackoutFinal-Web.pdf.

[3] S. Corsi and C. Sabelli, "General Blackout in Italy Sunday September 28, 2003, h. 03:28:00," in IEEE Power Engineering Society General Meeting, vol. 2, pp. 1691-1702, June 2004.

[4] "Preliminary Report into the Recent Electricity Transmission Faults affecting South London and East Birmingham," tech. rep., Office of Gas and Electricity Market, OFGEN, 30 September 2003.

[5] DIgSILENT PowerFactory Version 14.

[6] G. Benmouyal, M. Meisinger, J. Burnworth, W. Elmore, K. Freirich, P. Kotos, P. Leblanc, P. Lerley, J. McConnell, J. Mizener, J. P. de Sa, R. Ramaswami, M. Sachdev, W. Strang, J. Waldron, S. Watansiriroch, and S. Zocholl, "IEEE Standard inverse-time characteristic equations for overcurrent relays," IEEE Transactions on Power Delivery, vol. 14, pp. 868-872, 1999.

[7] North American Electric Reliability Corporation (NERC) hompepage, "Transmission relay loadability." http://www.nerc.com/files/PRC-0231.pdf.

[8] D. Hill, "Nonlinear dynamic load models with recovery for voltage stability studies," IEEE Transactions on Power Systems, vol. 8, pp. 166176, January 1993.

[9] H. Latorre, M. Ghandhari, and L. Söder, "Control of a VSC-HVDC Operating in Parallel with AC Transmission Lines," in IEEE PES Transmission and Distribution, Conference and Exposition, August 2006.

[10] K. Walve, "Modelling of power system components at severe disturbances," in CIGRÉ report 38-18, June 1986.

[11] M. Bahrman and B. Johnson, "The ABCs of HVDC transmission technologies," IEEE Power Energy Magazine, vol. 5, pp. 32-44, April 2007. 